\title{
¿Existen dinámicas regionales que generen pobreza?
}

Existe-t-il des dynamiques régionales qui engendrent de la pauvreté?

Are there regional dynamics that generate poverty?

\section{Evelyne Mesclier}

\section{(2) OpenEdition}

\section{Journals}

\section{Edición electrónica}

URL: http://journals.openedition.org/bifea/6818

DOI: $10.4000 /$ bifea. 6818

ISSN: 2076-5827

\section{Editor}

Institut Français d'Études Andines

Edición impresa

Fecha de publicación: 1 diciembre 2002

Paginación: 681-698

ISSN: 0303-7495

Referencia electrónica

Evelyne Mesclier, «¿Existen dinámicas regionales que generen pobreza? », Bulletin de l'Institut français d'études andines [En línea], 31 (3) | 2002, Publicado el 08 diciembre 2002, consultado el 08 diciembre 2020. URL : http://journals.openedition.org/bifea/6818; DOI : https://doi.org/10.4000/bifea.6818

\section{(c) (i) (9)}

Les contenus du Bulletin de l'Institut français d'études andines sont mis à disposition selon les termes de la licence Creative Commons Attribution - Pas d'Utilisation Commerciale - Pas de Modification 4.0 International. 


\title{
¿EXISTEN DINÁMICAS REGIONALES QUE GENEREN POBREZA?
}

\author{
Evelyne MESCLIER*
}

\section{Resumen}

En el año 2001 el Instituto Nacional de Estadística e Informática del Perú elaboró por primera vez un indicador de "pobreza monetaria" con cifras representativas para los departamentos. Ese indicador evalúa ya no la cobertura de servicios e infraestructuras en el lugar de residencia principal de los encuestados o las características de las familias y personas que las componen, sino su capacidad para generar ingresos. Los mapas que resultan son sorprendentes. Por ejemplo, la costa norte aparece como bastante pobre, pese a características generalmente consideradas como ventajosas, tales como los niveles bajos de fecundidad. Este artículo plantea la hipótesis de la existencia de dinámicas regionales — vinculadas con políticas y mercados nacionales y mundialesen las que la población se involucra a través de sus redes, las cuales relacionan territorios muy distintos entre sí: ciudades y campos, alturas y valles. Por lo tanto, los hogares pobres no lo son solamente como resultado de sus propias características o de las de su lugar de residencia principal, sino también porque estas dinámicas de mayor amplitud no tienen siempre, posiblemente debido al centralismo, efectos virtuosos e incluso pueden generar efectos perversos como la poca escolarización de los jóvenes.

Palabras claves: Perú, estadísticas, cartografía temática, pobreza, dinámicas regionales, movilidad.

\section{EXISTE-T-IL DES DYNAMIQUES RÉGIONALES QUI ENGENDRENT DE LA PAUVRETÉ ?}

\section{Résumé}

En 2001, pour la première fois, l'Institut National de Statistique et Informatique du Pérou a élaboré un indicateur de "pauvreté monétaire" avec des chiffres représentatifs au niveau des départements. Cet indicateur évalue non plus la présence de services et infrastructures dans le lieu

* Investigadora del IRD, UMR IRD/ENS “Territorios y mundialización en los países del sur”. E-mail: emesclier@yahoo.fr

Agradezco a Philippe Waniez por sus sugerencias. 
de résidence principal des familles de l'échantillon, ou les caractéristiques de celles-ci et des personnes qui les composent, mais leur capacité à générer des revenus. La cartographie de cet indicateur donne des résultats surprenants. Par exemple, la côte nord apparaît comme plutôt pauvre, malgré des caractéristiques généralement considérées comme avantageuses, comme le taux peu élevé de fécondité. On émet ici l'hypothèse de l'existence de dynamiques régionales — liées à des politiques et marchés nationaux et mondiaux - auxquelles participe la population à travers ses réseaux, qui relient des territoires très différents les uns des autres - villes et campagnes, hauteurs et vallées - . Ainsi, les foyers pauvres ne le sont pas seulement en raison de leurs propres caractéristiques ou de celles de leur lieu de résidence principale, mais aussi parce que ces dynamiques de plus grande ampleur n'ont pas toujours, peut-être à cause du centralisme, des effets vertueux, voire peuvent générer des effets pervers comme la faible scolarisation des jeunes.

Mots clés : Pérou, statistiques, cartographie thématique, pauvreté, dynamiques régionales, mobilité.

\title{
ARE THERE REGIONAL DYNAMICS THAT GENERATE POVERTY?
}

\begin{abstract}
In 2001, for first time, the Peruvian National Institute of Statistics and Informatics elaborated an indicator of "monetary poverty", with representative values at a departmental level. This indicator no longer evaluates the existence of services and infrastructures in the places of main residence of households, nor their characteristics and those of the individuals who make them up, but their capacity for generating income. Maps made on this basis are surprising. The North Coast, for instance, appears as quite "poor" despite of features generally considered as favorable, such as low fecundity rates. In this article, I suggest the existence of regional dynamics -connected to national and global policies and markets- in which people participate through their own networks linking very different territories — cities and countryside, highlands and lowlands-. Hence, poor households are poor not only because of characteristics of their own or of their main place of residence, but also because these regional dynamics do not always have perhaps due to centralism - virtuous effects and can even generate perverse ones, like low school attendance rates among young people.
\end{abstract}

Key words: Peru, statistics, thematic cartography, poverty, regional dynamics, mobility.

Los indicadores relacionados con las infraestructuras familiares o colectivas (estado de la vivienda, infraestructuras presentes en la localidad) se utilizan frecuentemente para estimar los niveles de pobreza de la población censada en un lugar determinado. Sin embargo, a menudo no se toma en consideración uno de los sesgos que conlleva ese método: en el Perú muchas familias e individuos utilizan alternativamente diversos puntos del territorio como lugar de vida y de trabajo. Sus redes familiares, sociales, laborales se extienden mucho más allá de los límites del distrito donde los encontraron los encuestadores. Sus pertenencias materiales no se limitan a las que poseen en ese único lugar. Por lo tanto, no se puede estimar el nivel de riqueza de una población únicamente a partir de los recursos materiales presentes en la localidad que corresponde a su residencia principal, sean éstos recursos colectivos o individuales. 
La posibilidad de representar cartográficamente la pobreza en base a los ingresos de los hogares (1)es de ese punto de vista un progreso, en la medida en que se evita juzgar el nivel de bienestar de las personas por el aspecto material del sitio donde residen sólo una parte del tiempo. Se estima el conjunto de riquezas que produjo ese hogar, cualquiera sea el sitio donde lo produjo. La comparación de esos ingresos con los ingresos necesarios para tener acceso a una canasta básica de consumo, aunque reintroduzca la necesidad de estimar el costo de esa canasta en el departamento al cual corresponde (2), es un indicador que toma en cuenta la movilidad y diversidad de actividades de las familias. Hasta la fecha no contábamos con ese indicador para los departamentos — sólo existía para conjuntos territoriales mayores-.

Los mapas (3) que resultan de ese ejercicio son sorprendentes, por diversas razones: no nos muestran la imagen clásica de una costa uniformemente más rica y una sierra y selva uniformemente más pobres. Es más, la heterogeneidad no aparece ahí donde uno la podría esperar: la costa sur es rica (4), pero la costa norte pobre; la sierra central es pobre, y Madre de Dios es rico. Queda demostrado que la calidad de los servicios locales o de la vivienda no tienen mucho que ver con los ingresos de las familias. Es más, si bien se verifica, tanto a nivel nacional como a nivel departamental, que la pobreza monetaria acompaña más a menudo a los bajos antes que a los altos niveles educativos y a la alta que a la baja fecundidad, esas relaciones parecen cambiar de amplitud según el departamento donde uno está, revelando un "efecto espacial" independiente de estos indicadores.

Esa repartición espacial parece abogar por la necesidad de describir los sistemas socio-espaciales que inervan el territorio nacional. Ese enfoque permite ir más allá de la constatación de una relación entre características de las familias y de su distrito o incluso departamento de residencia principal, y la probabilidad de que sean pobres. En efecto, estas características a su vez están relacionadas con historias económicas regionales que producen efectos a veces contradictorios y de cronologías complejas. Se trata de entender cómo las relaciones que construyen estos hogares en diferentes sitios de su ámbito regional tienen mayor o menor probabilidad de llevarlos a la pobreza que a cierto nivel de bienestar.

(1) Pobreza "monetaria", si bien los ingresos incluyen también el valor de los productos "autoconsumidos", lo cual constituye una mejora en comparación con las encuestas anteriores. Nos referiremos a lo largo de ese trabajo a los datos y análisis novedosos producidos por el INEI y sus colaboradores. Véase INEI, 2002; Herrera, 2002.

(2) Si es válida la hipótesis según la cual los espacios de vida de parte de los hogares van más allá de su departamento de residencia principal, es muy probable que realizen parte de sus gastos fuera de ese departamento. Estimar el costo de la canasta en totalidad en su departamento de residencia principal introduce por lo tanto un sesgo en el cálculo de la pobreza.

(3) Los mapas elaborados atribuyen a cada departamento las características, en términos de pobreza, de los hogares que tienen ahí su residencia principal, lo cual puede ser considerado también como un sesgo si se toma en cuenta que parte de las familias tal vez tengan una residencia secundaria en otro departamento.

(4) En términos del porcentaje de familias no pobres, y relativamente al resto del país, el cual es en ese texto el universo de referencia. 
Empezaré por mostrar que existen verdaderas "regiones" donde los indicadores relacionados con la pobreza tienen comportamientos a veces contradictorios, para luego describir los efectos de un boom económico regional particular, en el norte del país, y concluir finalmente con los posibles mecanismos de la generación de pobreza a nivel regional.

\section{LOS SORPRENDENTES RESULTADOS DE UNA NUEVA CARTOGRAFÍA}

\section{1. La importancia conceptual de poder representar cartográficamente la pobreza monetaria}

Los indicadores utilizados hasta ahora para comparar los niveles de vida de las familias en los distintos sitios del país medían mayormente la presencia o ausencia de diversos tipos de infraestructuras, colectivas y familiares, en el distrito donde esas familias habían sido censadas. No tomaban en cuenta, por lo tanto, los otros servicios a los cuales esas familias tienen acceso en el curso de sus desplazamientos, de corta, mediana o larga distancia. No estaba considerada por ejemplo la existencia de una segunda residencia en otro distrito, fenómeno sin embargo más frecuente que lo que generalmente se supone, a menudo vinculado a la existencia de redes familiares. Por otra parte estas infraestructuras se evalúan sobre la base de un "deseable" válido probablemente en Lima, mas no necesariamente en todas partes: para dar un ejemplo trivial, los materiales que se emplean en la construcción de la vivienda dependen por supuesto del nivel de ingresos de la familia, pero también del clima, de la presencia de recursos tales como piedras o madera en la zona, de la organización de la vida familiar y social en un sentido más amplio. Una cartografía de estos indicadores refleja por lo tanto mucho más que los niveles de vida de las familias; es decir, demasiado, si de medir la pobreza se trata (cf. Huerta et al., 1997).

Algunos indicadores, como la dependencia económica o el nivel de educación del jefe de familia, "siguen" con mayor facilidad a las familias en sus desplazamientos - aunque el hogar, tal como está descrito en el censo, pueda modificarse en el curso de éstos- La cartografía de estos indicadores, y particularmente del primero, revela sin embargo incoherencias: las tasas de dependencia económica resultan ser muy distintas entre distritos vecinos que comparten características muy parecidas en términos demográficos y económicos, lo cual llama la atención sobre la forma de definir el trabajo de la mujer (¿empleo u ocupación "su casa”?). La ambigüedad vale tanto en el caso de las mujeres campesinas como de las y los trabajadores familiares no remunerados, y al parecer no siempre llevó a los encuestadores al mismo tipo de opción. El nivel de educación de los jefes de hogar depende en parte de su edad: la cartografía por lo tanto señalará a las áreas del país con mayor proporción de personas mayores, cuando esta estructura de edades no tiene una relación clara con la pobreza.

La cartografía de las "necesidades básicas insatisfechas", mezcla de indicadores poco confiables y que no toman en cuenta que las actividades de un hogar pueden desarrollarse a distintas escalas, resultaba por lo tanto un ejercicio poco alentador. 
Los datos sobre pobreza monetaria elaborados por el INEI a nivel departamental permiten por lo tanto completar y mejorar las visiones anteriores de la diferenciación espacial en el país. El indicador es una medición directa de la cantidad de bienes que pueden adquirir las familias. Permite saber dónde están las familias pobres del país, y ya no solamente dónde están los sitios peor atendidos o incluso los sitios donde las características de las familias son las más desventajosas para su acceso a niveles de bienestar aceptables. La herramienta en este caso autoriza a percibir distintamente la relación entre espacio y pobreza, a eliminar las relaciones a priori que generaba el uso de indicadores indirectos. La cartografía de un indicador como el material de construcción de la vivienda conduce a una visión determinista de la pobreza (lleva a representar cartográficamente "regiones naturales" más que pobreza). La medición de la presencia o ausencia de servicios lleva también a una visión determinista, aunque ya no fundada sobre elementos naturales: la pobreza sería una emanación de "áreas pobres", más que el resultado de dinámicas sociales y económicas (5). Más directa, la medición de la intensidad de la pobreza a través de los ingresos permite abordar el tema sin haber definido primero una relación a priori entre una característica geográfica, sea esta ecológica o socioeconómica, y la pobreza misma.

Por supuesto el nivel departamental es muy grueso: es evidente la heterogeneidad de cada uno de los departamentos, tanto entre ciudades y campo como entre las subregiones económicas y a veces culturales que los componen. Es además una fuerte limitación metodológica para un análisis espacial de la situación, en cuanto la cantidad de observaciones localizadas, 24, es inferior al límite normalmente aceptable para un tratamiento cuantitativo de la información. Pese a esa falta de precisión, la cartografía obtenida resulta muy interesante, en tanto muestra una imagen muy distinta de la pobreza, la cual lleva a plantear nuevas hipótesis sobre cómo se genera.

\section{2. La hipótesis de la existencia de "efectos regionales" en la distribución de la pobreza}

El mapa de la pobreza elaborado por Herrera y el INEI(Fig. 1: Mapa 1) sorprende. Los mapas elaborados en base al censo de 1993, aunque a nivel más fino, mostraban una costa norte y una sierra central en tan buena situación como la costa sur en cuanto a muchos indicadores, de los cuales varios han sido señalados como relacionados con la probabilidad de ser pobre: niveles educativos, de urbanización, de fecundidad, de mortalidad infantil, en particular ( $C f$. Huerta et al., 1997). Si se abandona la clasificación "robusta" elaborada por Herrera (6) para adoptar una clasificación por sextiles (7) (Fig. 1: Mapa 2), tanto la costa norte como la sierra central aparecen en una situación intermedia entre la de los departamentos donde la proporción de pobres es baja y la de los departamentos donde es bastante alta.

(5) Para el debate sobre la relación teórica entre espacios y pobreza, véase en particular : Séchet, 1996.

(6) Herrera agrupa los departamentos en 5 clases que no son sensibles a los errores muestrales y toman en cuenta la existencia de diferencias no significativas entre los departamentos.

(7) Clasificación que comporta 6 clases de las cuales las 4 clases centrales cuentan con efectivos iguales y la primera y la última clase cuentan con un $5 \%$ de los individuos. 
Mapa 1: Mapa robusto de la Pobreza Total (tomado de J. Herrera, 2002)

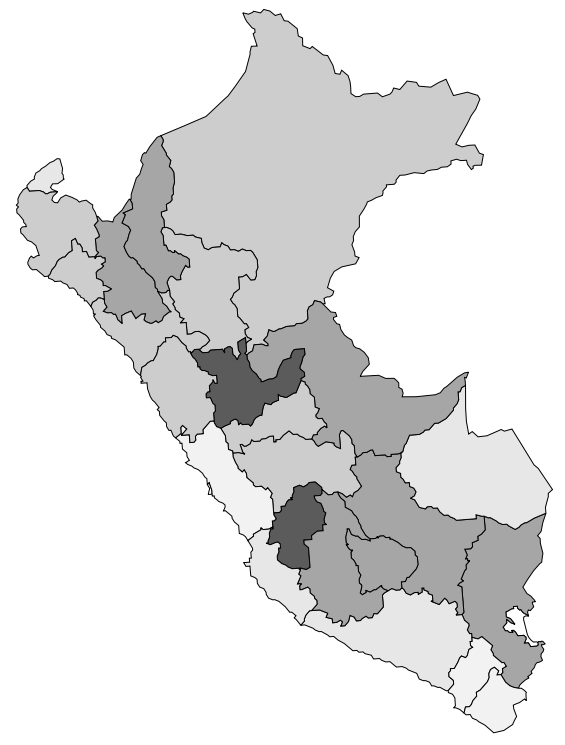

Rangos robustos de pobreza

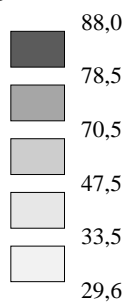

Mapa 2: Mapa de la pobreza, clasificación por sextiles

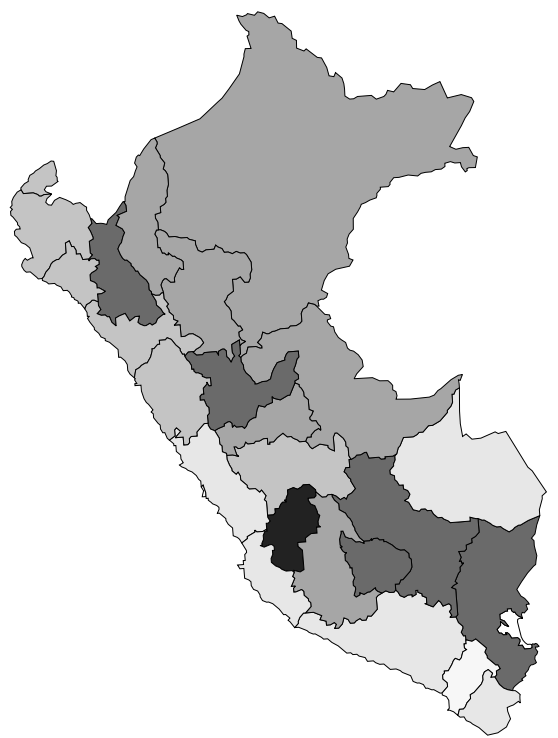

Porcentaje de hogares pobres

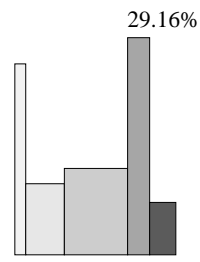

Fuente: INEI, Encuesta Nacional de Hogares IV trimestre 2001

Elaboración: E. Mesclier en base a Herrera, 2002 (mapa 1); E. Mesclier (mapa 2)

Software: Philcarto

Fig. 1 - Porcentaje de hogares pobres en 2001 a nivel departamental. 
Dos hipótesis podrían explicar la situación intermedia de esos departamentos: un cambio repentino (es decir, en un lapso de tiempo menor a diez años) en estos indicadores, para estas regiones, que podría deberse a las migraciones; un "efecto regional" que debilitaría localmente la relación entre educación, fecundidad, condición urbana por un lado, y pobreza por otro.

Resulta imposible sin embargo demostrar directamente la existencia de tal efecto regional. Los niveles de pobreza de cada departamento son totalmente independientes, en términos estadísticos, de los niveles de pobreza de los departamentos vecinos (8). En otras palabras, a esa escala no se observa, en forma general, una mayor semejanza entre departamentos vecinos en lo que concierne la pobreza. Los conjuntos espaciales que tienen niveles parecidos de pobreza están compuestos de pocos departamentos (dos o tres), de tal forma que su existencia podría ser el resultado tanto del azar como de una lógica espacial. Por otra parte, el departamento de Madre de Dios tiene un comportamiento muy distinto del de los departamentos vecinos y por el poco número de unidades su caso representa un peso importante en el cálculo de los niveles de autocorrelación espacial: esto contribuye a alejar los coeficientes de un valor que mostraría una relación entre comportamiento de los departamentos y vecindad.

Una vía indirecta para mostrar que ciertos grupos de departamentos tienen un comportamiento particular en relación con la pobreza consiste en observar cómo varían los niveles de pobreza en relación con diversos indicadores que sabemos están vinculados a nivel nacional con la pobreza. Sin embargo, por las mismas razones, resultará imposible demostrar que la existencia de pequeños grupos de departamentos que muestran comportamientos parecidos no es fruto del azar. No es sino la acumulación de estas observaciones que nos da pie para plantear la hipótesis de la existencia de efectos regionales, confortada por observaciones de campo. Nos parece sin embargo importante plantear esa hipótesis, pues de existir tales efectos, su consideración se volvería necesaria para entender cabalmente cómo se genera o se perpetúa la pobreza en el país.

\section{3. Pobreza y fecundidad, pobreza y educación: los comportamientos diferenciados de grupos de departamentos}

A nivel nacional pobreza y fecundidad, pobreza y niveles educativos aparecen como fuertemente relacionados: cuanto más niños tiene el hogar y tanto más bajos son los niveles educativos del jefe del hogar y de los otros miembros, mayor es la probabilidad que ese hogar sea pobre (INEI, 2002; Herrera, 2002). Aparece por lo tanto lógico buscar, como lo hace el INEI, una relación entre estos indicadores a nivel departamental. Nos interesaremos aquí no tanto por la existencia de esa relación, sino por la posición relativa de los departamentos frente a la relación promedio que existe a nivel departamental entre estos indicadores.

Fecundidad y pobreza a nivel departamental están relacionadas, comolo muestran los resultados de la regresión lineal, con un $\mathrm{R}^{2 \%}$ de $65 \%$. Sin embargo los mapas (Fig. 2) muestran tendencias regionales con cierta coherencia espacial: el norte, al cual

(8) Coeficiente de Moran -0,08; coeficiente de Geary 1,07. Cálculos realizados con el programa Philcarto (@ $\mathrm{Ph}$. Waniez). 
Mapa 1: Tasas de fecundidad

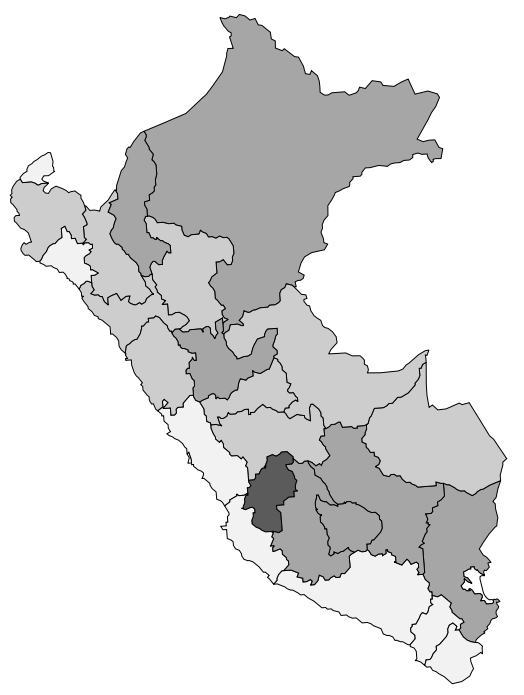

Número promedio de hijos por mujer

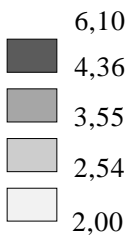

Mapa 2: Residuos de la regresión lineal pobreza versus fecundidad

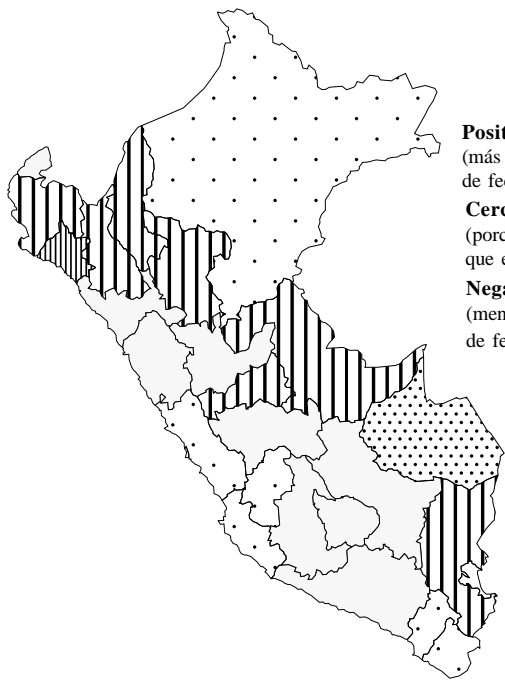

Residuos

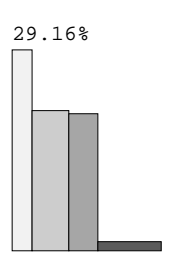

4,36

2,00

|l| || ||| más de una desviación estándar

\begin{tabular}{l|l|l|l|l} 
(más hogares pobres que lo que el nivel una desviación estándar \\
de fecundidad permite prever)
\end{tabular}

Cerca a cero que el nivel de fecundidad permite prever)

Negativos

(menos hogares pobres que lo que el nivel

de fecundidad permite prever)

$\mid$

menos de media desviación estándar

entre media y una desviación estándar

$\therefore$ más de una desviación estándar
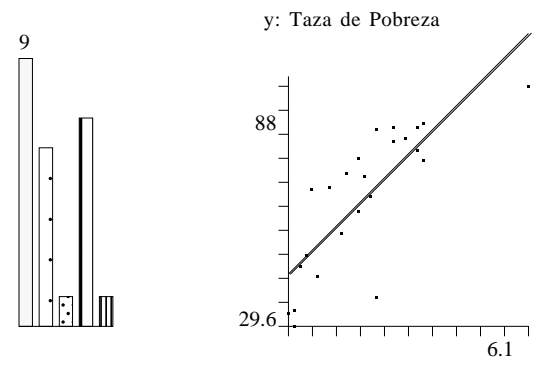

x: Fecundidad

Fuente: INEI, Encuesta Nacional de Hogares IV trimestre 2001

Elaboración: E. Mesclier

Software: Philcarto

Fig. 2 - Pobreza y fecundidad en 2001 a nivel departamental. 
se junta parte de la sierra y selva central, se caracteriza por una pobreza "exagerada" en comparación con los niveles de fecundidad; $y$, a la inversa, los departamentos de la costa sur (menos Arequipa) y Madre de Dios y Loreto son "ricos" pese a los niveles de fecundidad tanto o más elevados. El resultado no es muy distinto si se comparan niveles de fecundidad y rangos de pobreza tales como los definidos por Herrera para elaborar su mapa "robusto" de la pobreza.

La tasa de extrema pobreza departamental está más relacionada con la fecundidad departamental que la pobreza total, con un $\mathrm{R}^{2 \%}$ de $81 \%$. El mapa de los residuos muestra menos homogeneidad para el caso del norte, pero Lambayeque y Cajamarca, así como los departamentos de la sierra y de la selva central, tienen tasas de extrema pobreza ahí también "exageradas" en comparación con la fecundidad. Madre de Dios y algunos departamentos de la costa siguen mostrando niveles "bajos" de pobreza en relación con la recta de regresión.

La afirmación según la cual “ [...] el grado de pobreza de los departamentos tiene una vinculación directa con el lugar que ocupan en la transición demográfica [...]" (INEI, 2002: 30) merece ser relativizada por lo menos en el caso de la pobreza total - si bien el nivel de fecundidad global de la población no es un indicador suficiente para medir el avance de la transición demográfica-. Más interesante es constatar que se puede distinguir bloques regionales: la distribución de la variación probablemente no sea aleatoria en el espacio, aunque como ya se dijo resulte imposible cuantificar los efectos de autocorrelación espacial.

Tanto en el nivel departamental como en el conjunto nacional, la relación entre las tasas de pobreza y los niveles educativos es fuerte: para las 24 unidades espaciales, $\mathrm{R}^{2} \%$ es igual a $72 \%$ para la regresión lineal entre la tasa de pobreza y el porcentaje de bajos niveles educativos (hasta primaria completa) y a $79 \%$ en el caso de la extrema pobreza. La relación es más débil entre las tasas de pobreza y los niveles de educación superior. Herrera (2002: 36) resalta la importancia de los niveles de educación del jefe del hogar y de las personas del hogar en conjunto, en la probabilidad de una familia de ser pobre, pero también la complejidad de la relación entre estas variables, según el contexto urbano o rural en particular.

Los datos reflejan cierta lógica espacial, aunque no tan evidente como en el caso de la relación entre pobreza y fecundidad. El norte, con excepción del departamento de Lambayeque, tiende a ser menos pobre que los niveles de educación de las personas dejarían esperar. Es el caso también del extremo sur (Tacna, Moquegua), de Ayacucho y de Madre de Dios (Fig. 3: Mapa 2).

Así, mientras los departamentos del norte son más pobres que lo que dejan prever las tasas de fecundidad, pero menos pobres que lo que dejarían prever los niveles de educación (con excepción de Lambayeque), en Madre de Dios, Moquegua o Tacna, los niveles de pobreza son más bajos que lo que dejarían prever las tasas de fecundidad y los niveles educativos. En la sierra y selva centrales, así como en Puno y en Lambayeque, la pobreza es mayor que lo que deja esperar la fecundidad y mayor que lo que dejan esperar los niveles de educación.

Finalmente, la pobreza total y la extrema pobreza a nivel de los departamentos están también fuertemente vinculadas $\left(\mathrm{R}^{2} \%=88 \%\right)$, y resalta nuevamente la particularidad 
Mapa 1: Población de 25 y más años de edad sin estudios secundarios
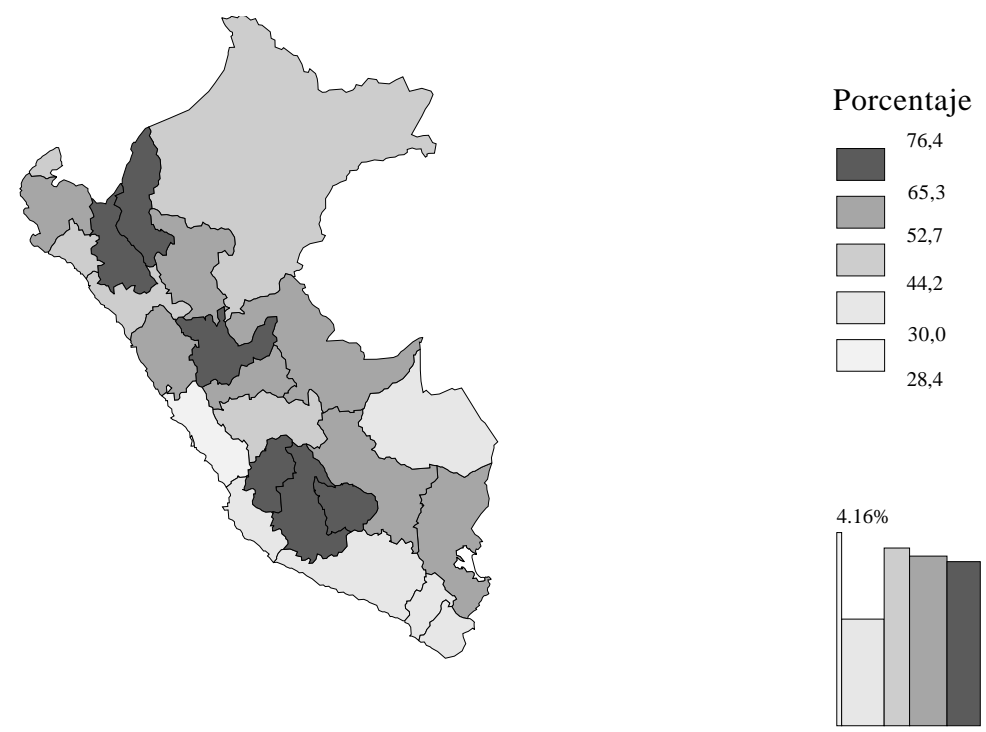

Mapa 2: Residuos de la regresión lineal pobreza versus porcentaje de personas sin estudios secundarios

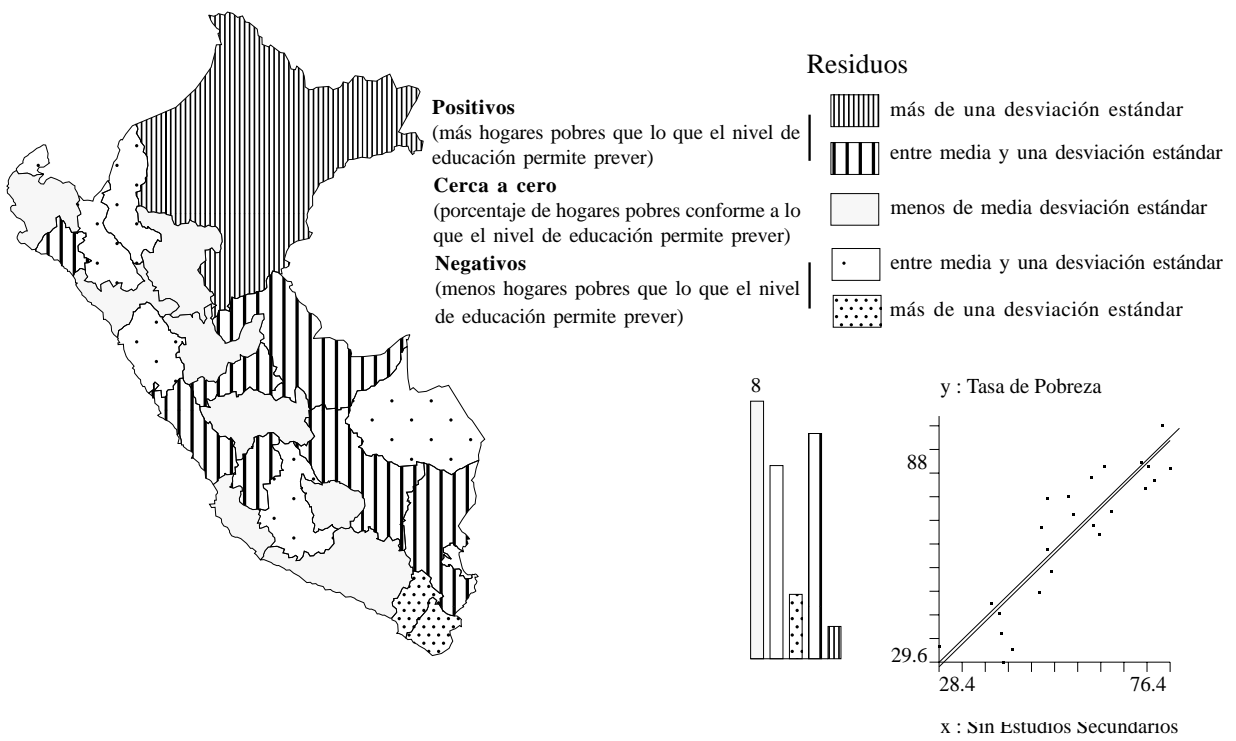

Fuente: INEI, Encuesta Nacional de Hogares IV trimestre 2001 Elaboración: E. Mesclier Software: Philcarto

Fig. 3 - Pobreza y educación en 2001 a nivel departamental. 
del norte (La Libertad, Lambayeque, Piura, Tumbes, Amazonas), como bloque regional caracterizado por una "extrema pobreza" que resulta ser bastante baja, en comparación con los niveles de pobreza (Fig. 4).

La poca cantidad de observaciones limita mucho, como se dijo, el interés de estos resultados: las relaciones están estimadas con un número pequeño de unidades espaciales, por lo cual es difícil considerar que las diferencias tengan mucha significación. La repartición de los residuos permite pensar que la regresión lineal no es la mejor aproximación a la relación entre las variables consideradas. Sin embargo, los departamentos del norte (Cajamarca, Amazonas, Piura, Lambayeque, San Martín, La Libertad) se diferencian claramente de los otros departamentos.

¿Cómo explicar que los departamentos que comparten la misma distancia del modelo nacional sean precisamente departamentos vecinos, cuando por otra parte notienen características similares en cuanto a ecología, producción, urbanización? ¿Cómo explicar que la disminución de la fecundidad no esté asociada, ni en el norte, ni en la sierra central, a una disminución de la pobreza comparable a la que se observa en la costa sur o en Madre de Dios? ¿Cómo explicarque los buenos niveles educativos alcanzados en Lambayeque por un lado, la sierra y selva central por otro, asociados a una fecundidad baja, no hayan permitido a la población de estos departamentos reducir significativamente sus niveles de pobreza? Trataremos de mostrar que parte de la respuesta radica en la existencia de redes espaciales a nivel regional, basándonos en la historia reciente de la región norte.

\section{BOOMS ECONÓMICOS, REDES ESPACIALES Y GENERACIÓN DE POBREZA}

Hasta mediados de los años 90 , el eje que vincula a la selva norte con la costa norte y Chiclayo parecía ser uno de los más dinámicos, sino el más dinámico, de todo el país. Esto se debía en gran parte al auge de la producción de coca, que empezó en los años 80 en el Alto Huallaga (en el límite de los departamentos de Húanuco, San Martín, Ucayali, La Libertad y Loreto) para extenderse hacia Ucayali (Aguaytía) y el bajo Huallaga (es decir, el piedemonte de los Andes cajamarquinos) hacia los años 19931994 (Bourliaud et al., 1998, 120).

\section{1. Constitución de una macrorregión alrededor de la producción de la coca}

Aunque sin ser comparables a los flujos migratorios hacia Lima, los flujos migratorios "definitivos" (9) hacia el río Huallaga, como hacia el Ucayali, eran considerables cuando el censo de 1993 permitió medirlos. Una cartografía de estos flujos (Huerta et al., 1997: 63) desdibujaba en ese entonces cuatro grandes tipos de procedencia para el Huallaga: la región de Tingo María, los Andes en el norte de Cajamarca y el oeste de Amazonas, las ciudades regionales (Chiclayo, Trujillo,

(9) En el caso del censo de 1993, se considera como migración definitiva un cambio de residencia entre 1988 (lugar donde la persona declara haber residido) y 1993 (lugar donde la persona fue censada). 
Mapa 1: La extrema pobreza

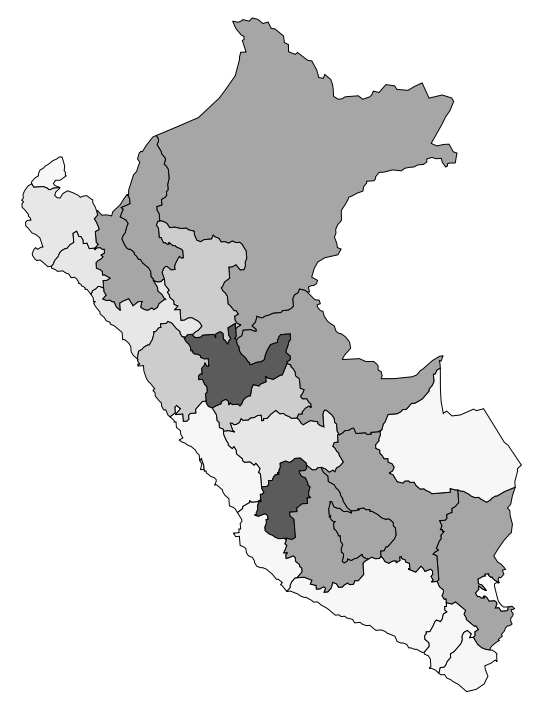

Porcentaje de hogares en situación de extrema pobreza
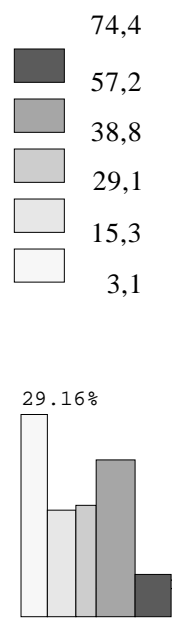

Mapa 2: Residuos de la regresión lineal extrema pobreza versus pobreza

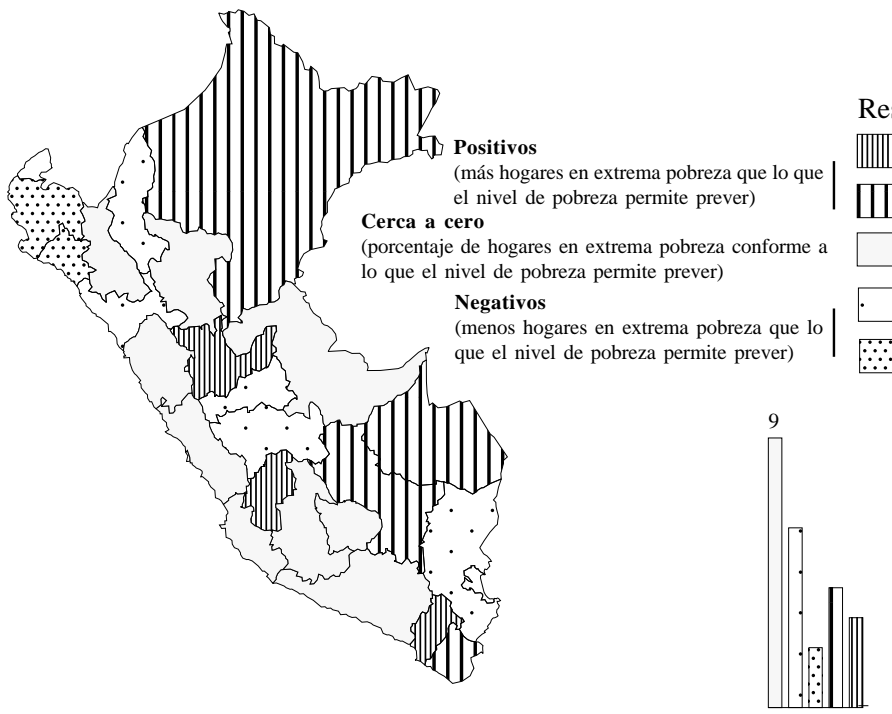

Fuente: INEI, Encuesta Nacional de Hogares IV trimestre 2001 Elaboración: E. Mesclier Software: Philcarto

\section{Residuos}

|||||||||| más de una desviación estándar

$\prod$ entre media y una desviación estándar

menos de media desviación estándar . entre media y una desviación estándar $\because \because 6$ más de una desviación estándar

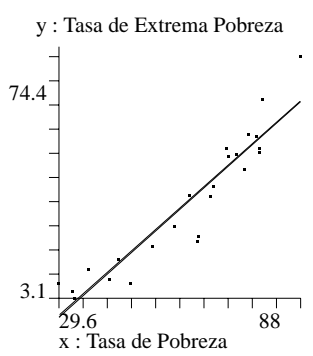

Fig. 4 - Pobreza y extrema pobreza en 2001 a nivel departamental. 
Huánuco, Iquitos), y Lima. Hacia la región del Aguaytía llegaban migrantes desde Lima también, y mayormente desde las provincias del departamento de Huánuco.

La intensidad de las redes espaciales que se formaron o se reforzaron en ese entonces no se puede medir sin embargo solamente a partir de las migraciones definitivas, pues muchas personas migraban hacia estas regiones en forma tan sólo temporal, entre dos tareas en sus propias chacras. Dicha migración no era perceptible en un aumento de las tasas de actividad de los adultos en las áreas de procedencia, pues estos adultos estaban en su mayoría ya en actividad y complementaban sus ingresos mediante la migración temporal.

La actividad específica generada por el boom de la coca en la región se aprecia en cambio en las tasas excepcionalmente elevadas de la actividad laboral de los adolescentes. Los datos del censo de 1993 permiten representar cartográficamente la tasa de actividad de los niños entre 6 y 14 años: el mapa a nivel provincial (Fig. 5) muestra una nítida acentuación del trabajo de los niños en dos regiones, el norte en la cordillera y la vertiente amazónica y el sur a proximidad de la vertiente amazónica y de la región de extracción de oro de Madre de Dios. Los coeficientes de autocorrelación espacial confirman la existencia de efectos regionales en la distribución espacial de la actividad de los niños.

En base a trabajos de campo realizados en el norte del Perú (10), aparece evidente la vinculación de ese boom con el financiamiento de otras actividades de tipo agrícola o comercial: cultivo de arrozen los valles de la vertiente occidental de los Andes, compra de tierras en el piedemonte, creación de comercios en las ciudades.

\section{2. Los efectos empobrecedores del boom: déficit de educación de los ex trabajadores, contracción brutal del capital en la región}

Más revelador aún del peso del trabajo de los jóvenes, no solamente directamente en la producción de coca, sino también en la renovación de la actividad regional, la baja tasa de escolaridad de los adolescentes (entre 12 y 17 años) era en 1993 un fenómeno de obvia relación con una dinámica regional (Fig. 6).

No sorprende por lo tanto finalmente que los departamentos del norte se caractericen por niveles educativos más bajos que, por ejemplo, en la costa sur. En comparación con la cartografía de los años 90 , donde no se apreciaba una gran diferencia en el progreso de la educación entre el sur y el norte, en el 2002 la diferencia es importante. Pero tampoco es sorprendente que estos bajos niveles educativos no estén acompañados de una pobreza aún mayor: la poca incidencia de la extrema pobreza, en particular, probablemente refleje el hecho que la región entró en crisis desde hace solamente algunos años.

La política impuesta por el Fondo Monetario Internacional, en cuanto a reducción de las superficies sembradas con coca, ha sido escrupulosamente aplicada en la región por el gobierno del ex presidente Fujimori. La recesión regional generada por la pérdida

(10) En el marco de una investigación colectiva sobre la reconfiguración de los espacios rurales en los Andes. 


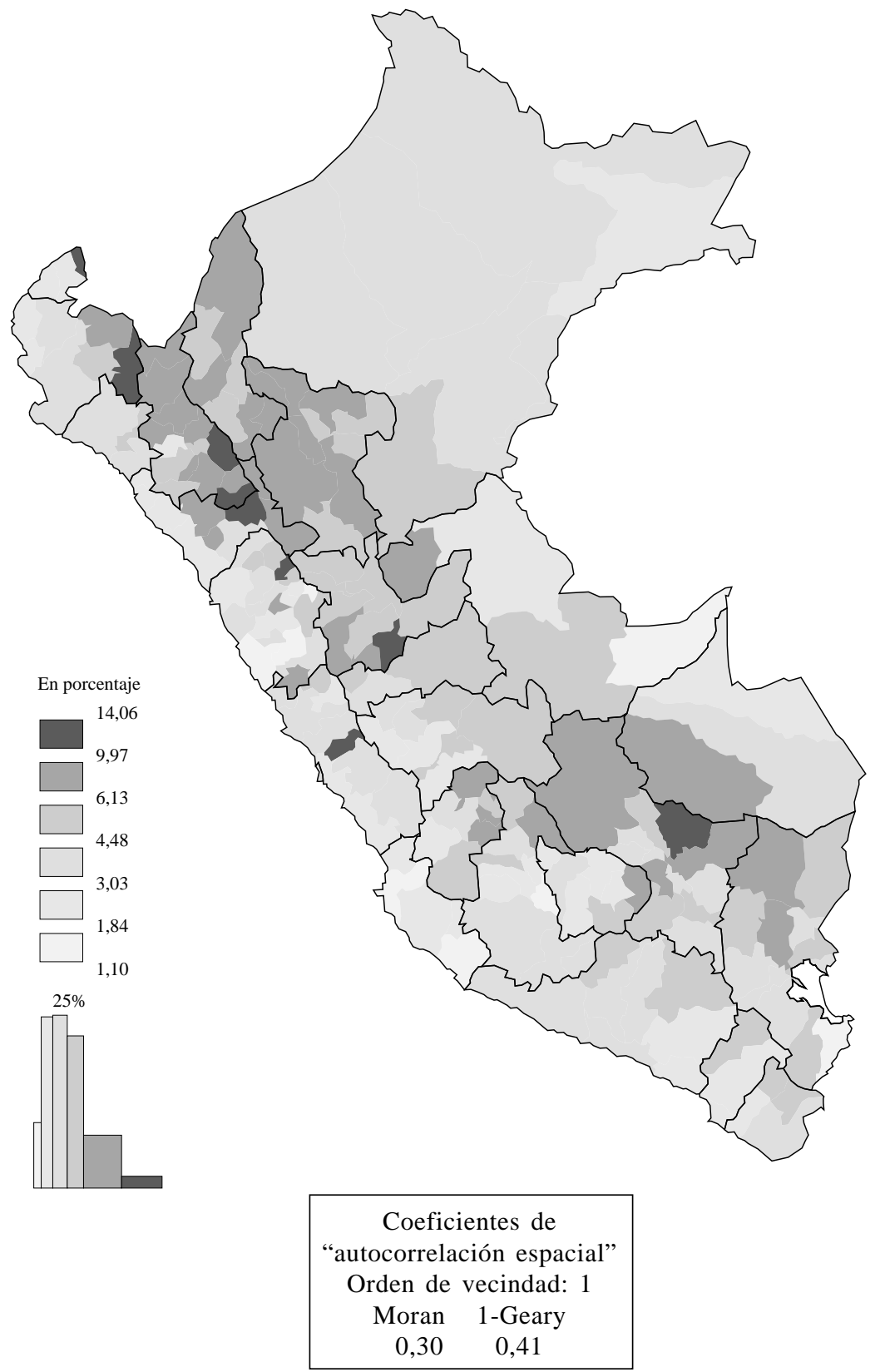

Fuente: INEI, Censos nacionales de población y vivienda 1993 Elaboración: E. Mesclier Software: Philcarto

Fig. 5 - Tasa de actividad de los niños de 6 a 14 años en 1993 a nivel provincial. 


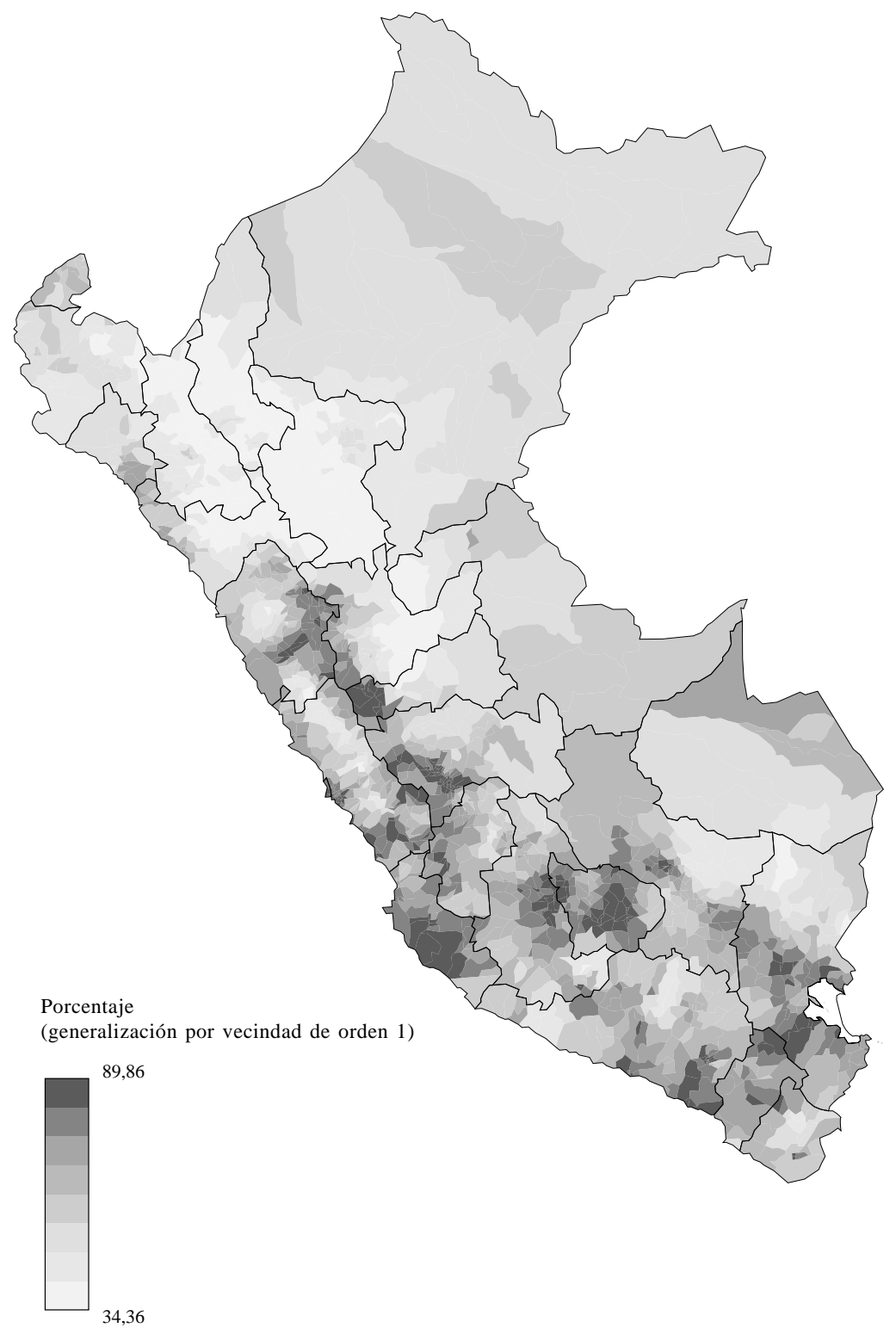

Fuente: INEI, Censos nacionales de población y vivienda 1993 Elaboración: E. Mesclier

Software: Philcarto

Fig. 6 - Tasa de asistencia escolar de los niños entre 12 y 17 años en 1993 (generalización espacial de orden 1 a nivel de los distritos). 
de esa fuente de ingresos y capitales, refuerza los efectos de la crisis de escala nacional que se precisa a partir de 1998 y los de la caída del precio del café a nivel internacional.

Los efectos colectivos de esa recesión no conciernen solamente las actividades productivas que ya no están financiadas. La falta general de dinero impide por ejemplo que los jóvenes del campo puedan ser acogidos en las casas de familiares en las ciudades para proseguir con sus estudios, como algunos estaban acostumbrados a hacer en años anteriores. Se vuelve difícil para las jóvenes conseguir un empleo de doméstica en las ciudades regionales. La disminución de la circulación de capital entorpece las relaciones económicas incluso en las necesidades más limitadas, como la compra de baterías para ver televisión o la posibilidad de conseguir un préstamo informal en caso de necesidad.

\section{3. La amplitud del fenómeno de constitución de espacios regionales en el Perú}

El hecho que muchos individuos y familias utilizan alternativamente diversos puntos del territorio como lugar de vida y de trabajo y son propensos a participar en la creación de dinámicas macrorregionales es a la vez conocido y muy difícil de medir. Las migraciones "definitivas" de los peruanos aparecen en los censos de población bajo dos formas: el cambio de residencia entre el momento del nacimiento y el momento del censo, y el cambio de residencia entre el momento del censo y 5 años atrás. Las migraciones temporales pocas veces han sido cifradas mediante encuestas. Sin embargo, existen otras modalidades de la movilidad que los estudios monográficos mencionan, pero que no son objetos de medición estadística. Se trata de un fénomeno importante que no se reduce a la migración, pues, como lo subraya Alber (1999: 188), la “[...] existencia de una red de reproducción que vincula unidades residenciales en distintos lugares [...]" implica que "[...] los movimientos entre estas unidades residenciales tienen que ser descritos dentro de este contexto y no como distintos tipos de migración temporal o permanente". Para Alber, sería "[...] adecuado reflexionar acerca del entrelazamiento de unidades residenciales, la fluctuación humana y el flujo de capital entre unidades residenciales, al igual que tematizar los cambios socioeconómicos de regiones enteras causados por estos procesos" (Alber, 1999: 190).

Poco se ha publicado en el Perú sobre los fenómenos de "oscilación” y de "residencia en dos unidades residenciales", no necesariamente porque se trate de "nuevas formas de vida" como lo piensa Alber (1999: 190), sino tal vez porque no se ha prestado mucha atención a estos rasgos que de ninguna manera eran compatibles con la imagen de campesinado "tradicional” que se atribuyó a la mayor parte de la población del país durante mucho tiempo.

Los estudios de campo que pude realizar en distintas partes del Perú (en la ex "región Inka", en la sierra central, en la región de Chiclayo) me llevan a matizar la imagen de una población rural sistemáticamente envuelta en formas de vida multiresidenciales y, a la vez, a dudar de la aparición de ese tipo de movilidad como forma de vida solamente en las últimas décadas. Limitándonos al período contemporáneo, se puede sugerir que muchos individuos fueron siempre muy móviles a menudo por razones de trabajo, que implicaba su ausencia durante parte del año —o parte del mes, o parte de la semana- del hogar principal y su presencia en un lugar donde también 
tenían de una $\mathrm{u}$ otra forma residencia habitual, mientras otros se volvieron menos móviles o cambiaron de tipo de movilidad en el curso de su vida, debido a razones como la adquisición de parcelas de tierra en el curso del proceso de Reforma Agraria o, en el mismo proceso, la pérdida del acceso a recursos ubicados en distintos sitios. Otros sin duda se volvieron más móviles cuando se aceleró la urbanización y sus hijos se instalaron en las ciudades, pues no deseaban — por razones de preferencia personalo no podían - por razones económicas - migrar definitivamente. Los contextos regionales y locales, en sus aspectos históricos, influyen probablemente mucho en la configuración de las actuales redes, en su amplitud, en la generalización de la movilidad de los pobladores.

Los fenómenos de “oscilación”, más aún que las migraciones, hacen muy probable que las dificultades económicas se difundan rápidamente entre todos los lugares incluidos en estas redes, incidiendo en la pobreza más que los propios recursos locales. Lima es a priori uno de los puntos importantes de estas redes, pero el caso del norte muestra que existen también dinámicas más propiamente regionales. Sería importante conocer más acerca de estas redes, de una forma más directa que la que tratamos de emplear aquí — lo que las encuestas del INEI tal vez hagan posible pronto-.

\section{CONCLUSIÓN: LA GENERACIÓN DE POBREZA EN LAS REGIONES}

La pobreza no es una característica permanente de las familias y tampoco una condición determinada por su lugar de residencia principal. En el caso de Lima, Herrera demostró que entre 1990 y 1994 la mitad de las familias pobres habían salido de esa condición (Herrera, 2000: 91). Por cierto, se trata de un periodo muy particular de la historia económica peruana y la mitad de estas familias eran de nuevo pobres en 1996. Si las familias no son permanentemente pobres, parece poco razonable pensar en la pobreza como un efecto de sus características propias o de las condiciones materiales y culturales locales, las cuales no se modifican con tanta velocidad, salvo excepción.

En cambio, apoyándonos en los elementos presentados, podemos plantear la hipótesis que existen dinámicas regionales independientes de la dinámica nacional, que pueden generar tanto una riqueza excepcional como una caída a niveles de pobreza peores que en el pasado. Obviamente estos fenómenos no están desconectados de otros que ocurran a nivel nacional o global — es el caso de la producción de coca- pero tienen una inscripción territorial regional.

No se trata de negar la influencia de la estructuración espacial del conjunto nacional en la pobreza, tal como la plantea Béjar, para quien "cuando se examina el mapa geográfico del Perú, es fácil comprobar que las zonas más pobres, donde los niveles de desnutrición son mayores, son las más alejadas de la capital y aquellas donde la población no tiene acceso al poder político" (Béjar, 2001: 157). Sin embargo la relación, en cuanto a pobreza monetaria, no parece ser tan directa, desde lo nacional hasta lo local, sino que implica fenómenos de escala regional o macrorregional.

Lima por supuesto no está excluida de los lugares que forman parte de las redes espaciales de los peruanos. Si bien existen dinámicas regionales que no se extienden al 
conjunto nacional, como las que intenté describir para el norte, el centralismo parece impedir que se genere una dinámica de desarrollo sostenido en las regiones. Entre 1988 y 1993, tan sólo la ciudad de Arequipa atrajo una mayor o igual cantidad de inmigrantes que Lima en un ámbito grande, conformado por los departamentos de Puno, Tacna, Moquegua, el sur del departamento de Cusco y el departamento de Arequipa mismo (Huerta et al., 1997: 61). En cambio, pese a la dinámica generada por el boom de la coca, la población del norte del país seguía migrando mayormente hacia la capital nacional (Huerta et al., 1997: 61). Salvo en el caso de Arequipa, los movimientos de escala nacional dominan sobre las redes regionales, por lo menos en el caso de ese tipo de migraciones consideradas como "definitivas" y muy probablemente en el caso de las inversiones que realizan las familias de origen provinciano.

Un boom regional provoca por lo tanto efectos regionales importantes, pero momentáneos si no genera una suficiente inversión a nivel local, y a veces hasta perversos: en el norte peruano parece haberse suspendido el esfuerzo de los jóvenes y sus familias para mejorar sus niveles educativos en cuanto podían emplearse en actividades lucrativas en la región. Más difícil será ahora para ellos conseguir un empleo en las ciudades regionales o en Lima.

Existe en este caso, y en otros, una verdadera dinámica regional, sobre la cual convendría reflexionar con mayor detenimiento, tanto para comprender la pobreza, como tal vez para imaginar soluciones para eliminarla. Sería interesante tomar en cuenta la participación de individuos y familias, pobres y no pobres, en las redes que vinculan territorios de características diversas entre sí, sobre la base de una cercanía relativa, de relaciones previas, de la posibilidad de vender mano de obra, de invertir capitales, para entender cómo se escapa ¿o desplaza? la riqueza y se regenera una y otra vez la pobreza.

\section{Referencias citadas}

ALBER, E., 1999 - ¿Migración o movilidad en Huayopampa? Nuevos temas y tendencias en la discusión sobre la comunidad campesina en los Andes, 213p.; Lima: IEP.

BÉJAR, H., 2001 - Política social, justicia social, 289p.; Lima: Cedep.

BOURLIAUD, J., DOLLFUS, O. \& GONDARD, P., 1998 - Pérou: le Haut-Huallaga, de la coca à l'abandon. Problèmes d'Amérique Latine, $\mathbf{N}^{\circ} 28$ : 109-123

HERRERA, J., 2000 - Ajustement et mobilité économique à Lima. Problèmes d'Amérique Latine, $\mathbf{N}^{\circ} 38$, juillet-septembre 2000: 71-99.

HERRERA, J., 2002 - La Pobreza en el Perú en 2001. Una visión departamental, 176p.; Lima: INEI-IRD.

HUERTA, L., MATEO, D. \& MESCLIER, E., 1997 - Perú en Mapas. Atlas en base al censo de población y vivienda, 136p.; Lima: INEI-ORSTOM.

INEI, 2002 - Condiciones de vida en los departamentos del Perú, 2001, 327p.; Lima.

SÉCHET, R., 1996 - Espaces et pauvretés. La géographie interrogée, 213p.; Paris/Montréal: L'Harmattan. 